\title{
Social Advantage with Mixed Entangled States
}

\author{
Aritra Das', Pratyusha Chowdhury ${ }^{2}$ \\ ${ }^{1}$ Department of Physics, Indian Institute of Technology Kanpur, Uttar Pradesh, India \\ ${ }^{2}$ Department of Physics, St. Xavier's College, Kolkata, West Bengal, India \\ Email: aritrab@iitk.ac.in, pratyusha.chowdhury03@gmail.com
}

How to cite this paper: Das, A. and Chowdhury, P. (2020) Social Advantage with Mixed Entangled States. Journal of Quantum Information Science, 10, 11-22. https://doi.org/10.4236/jqis.2020.102002

Received: March 2, 2020

Accepted: April 23, 2020

Published: April 26, 2020

Copyright $\odot 2020$ by author(s) and Scientific Research Publishing Inc. This work is licensed under the Creative Commons Attribution International License (CC BY 4.0).

http://creativecommons.org/licenses/by/4.0/ (c) (i) Open Access

\begin{abstract}
It has been extensively shown in past literature that Bayesian game theory and quantum non-locality have strong ties between them. Pure entangled states have been used, in both common and conflict interest games, to gain advantageous payoffs, both at the individual and social level. In this paper, we construct a game for a mixed entangled state such that this state gives higher payoffs than classically possible, both at the individual level and the social level. Also, we use the I-3322 inequality so that states that aren't useful advice for the Bell- $\mathrm{CHSH}^{1}$ inequality can also be used. Finally, the measurement setting we use is a restricted social welfare strategy (given this particular state).
\end{abstract}

\section{Keywords}

Non-Local Games, Mixed Entangled States, Social-Welfare-Solution

\section{Introduction}

Quantum theory emerged when most physicists realized that physics at the atomic level could not be completely described by classical mechanics. Planck was the first to propose the notion of "quanta", which was further developed by Einstein. Though Heisenberg and Bohr, the further luminaries of the theory believed in the innate uncertainty in the behavior of atoms, Einstein never accepted it. He fundamentally opposed the Copenhagen interpretation of Quantum Mechanics (QM). Thus Einstein-Podolsky-Rosen (EPR) put forward the EPR paradox in their paper in 1935 [1], which claimed QM was incomplete, that is, it did not provide a complete picture of our physical reality.

To resolve this, in their paper, Einstein-Podolsky-Rosen argued for the inclusion of a Hidden Variable Theory which would remove all the indeterminism in QM. This led Bohr to publish a paper in the same journal, under the same name, ${ }^{1}$ Bell-Clauser-Horne-Shimony-Holt inequality, 1969. 
where he stated that the criterion of physical reality given by EPR contains an essential ambiguity when applied to quantum phenomenon [2]. Hence there continued a debate between Einstein and Bohr regarding the fundamental nature of reality.

In 1964, Bell formulated an inequality [3] which was satisfied by all local realistic theories. Eventually, the quantum violation of Bell's inequality proved that no local realistic hidden variable Theory can exist from which QM can be derived. The value by which QM violates a particular Bell inequality is called the Tsirelson bound for that particular Bell Inequality. The Tsirelson bound for Bell-CHSH [4] is $2 \sqrt{2}$.

Quantum states that violate Bell's inequality are all non-local states (entangled). However, this leads to the question:

Is Bell's inequality sufficient to show non-locality (or entanglement)?

It turns out that Bell's inequality is not sufficient to prove non-locality. The states that violate Bell's inequality are definitely non-local, but there are other states that do not violate a particular Bell inequality but are still non-local. For example, the mixed entangled state in Equation (5) that we consider as the shared resource, does not violate the Bell-CHSH inequality. However, the state is still non-local as can be seen from its violation of the I-3322 inequality. This violation is due to the fact that the I-3322 inequality [5] is inequivalent to any $\mathrm{CHSH}-$ like inequality and thus may be used to detect such non-separable states that are not witnessed by any $\mathrm{CHSH}$-like inequality.

By constructing a proof-of-principle non-local game using this inequality and this particular mixed entangled state, we demonstrate that non-pure but nonseparable states are also useful as quantum advice (QSWA, defined in Sec. 3.4). By contrast, we know that any two-qubit pure entangled state can be used as QSWA in some non-local game. This result has interesting implications for quantum game theory in general, and quantum cryptographic protocols in particular. The utility of arbitrary (undistillable) bound entangled states in this context, however, remains an open problem that requires further study.

\section{Game Theory}

Game theory is mathematical modeling of strategic interaction among rational beings, used widely in economics [6], political sciences [7], biological phenomena [8], as well as logic, computer science and psychology [9]. It is the study of human conflict and cooperation, or in other words the study of optimal decision making of different players, each with a set of action having particular payoffs. It is the payoff which decides the preference of an action over another. Von Neumann and Morgenstern [10] were the pioneers of game theory.

Games can be cooperative (common interest) or non-cooperative (conflict interest). Cooperative games include competition between groups whereas non-cooperative game includes analyzing strategies and payoffs of individual players using the concept of Nash equilibrium [11]. In a game, if a player chooses a unique action 
from a set of available action it is called pure strategy, but if a probability distribution over a set of action is available it is called mixed strategy. Nash proved that in any game with finite number of action for each player there is always a mixed strategy Nash equilibrium. Later the concept of Bayesian games i.e., games of incomplete information was introduced [12] and Aumann proved the existence of correlated equilibria [13] in these games, as opposed to Nash equilibria.

\section{Quantum Game Theory}

\subsection{Non-Locality and Bayesian Game Theory}

Non-locality is one of the most counterintuitive aspects of QM. The principle of locality states that an object can be instantaneously affected only by its immediate surroundings and not remote or distant objects. However, quantum theory is not consistent with this and is inherently non-local in nature, unlike the rest of classical physics. For example, two entangled particles placed far apart, can display correlations in simultaneous measurements inexplicable in classical physics. These correlations can not be a result of a signal transfer as that would imply superluminal communication whereas in 1964 Bell [3] showed that they could not arise from predetermined strategies either.

In 2013, Brunner and Linden [14] demonstrated strong links between quantum non-locality and Bayesian game theory. Specifically, they showed that the normal form of a Bayesian game is equivalent to a Bell inequality test scenario. They showed that when the two players in the game share non-local resources such as an entangled pair of quantum particles, they can outperform players using any sort of classical resources. This can happen, for example, when the payoff function of the players corresponds to a Bell inequality, like the CHSH inequality [4], as first discussed by Cheon and Iqbal [15] but also when the payoff function doesn't correspond to any Bell inequality. They showed that more generally, for Bayesian games, QM provides a clear and indisputable advantage over all classical resources.

\subsection{Non-Locality in Conflict Interest Games}

Brunner and Linden showed that QM indeed provides an advantage over classical resources for all Bayesian games, but the examples they provided were all common interest games (games where it is beneficial for both the players to cooperate rather than oppose each other). In fact, until 2012, all other known non-local games, including the GHZ-Mermin game [16], the Bell-CHSH game [4], and the hidden matching game [17] [18] were all examples of common interest games (mostly, because the average payoff functions for both Alice and Bob were the same).

In 2012, $\mathrm{Zu}$ et al. [19] proposed a zero-sum (conflict) game where a player using proper quantum strategies could always win. However, for zero-sum games, all strategies are Pareto optimal, meaning, the sum of payoffs is the same for all strategies. In 2015, Anna Pappa et al. [20] demonstrated that quantum 
advice can offer an advantage compared to classical advice even in conflicting interest games. They explicitly constructed an incomplete information game with conflicting interests, where quantum strategies yielded fair equilibria with average payoffs strictly higher than those achievable by classical means, for both the players. Hence, Anna Pappa's work [ibid] was the first where the sum of payoffs was being increased above the classical maximum by using quantum strategies.

\subsection{Fair and Unfair Strategies}

Classical equilibria can be of two types:

- Fair equilibria, where the average payoffs for both the players are equal; and

- Unfair equilibria, where the payoffs for the players are unequal.

Up until 2016, most of the games (both common interest and conflicting interest) proposed, dealt with fair equilibria-that is, they showed that quantum fair payoffs surpass classical fair equilibrium payoffs. In 2016, Roy et al. [21] showed that quantum strategies can outperform not only fair classical equilibrium strategies but unfair strategies too. They analytically characterized some non-local correlations, that would yield unfair average payoffs strictly higher than the classical ones in Anna Pappa's game.

\subsection{Social Welfare Solutions and Pure Entangled States}

Until now, we've been concerned only with equilibria for individual playersstates where the players can't increase their payoffs further by unilaterally changing their individual strategies. Such equilibria are called correlated equilibria (as opposed to Nash equilibria). Psychological factors indicate that sometimes, instead of focusing solely on their individual payoffs, players may also consider additional social goals-one such idea is the Social Welfare Solution (SWS). In such a strategy, players aim to maximize the sum of their individual payoffs. Out of all the possible quantum strategies, the ones that increase the sum of the payoffs (above the classical value) are called Quantum Social Welfare Solution (QSWS) and the quantum state producing this strategy is called Quantum Social Welfare Advice (QSWA).

In 2019, Banik et al. [22] showed that any two-qubit pure entangled state can act as QSWA for some Bayesian game. Hence given any pure entangled state between two qubits, there exists at least one game where this state provides QSWS.

\section{Mixed Entangled States and the I-3322 Inequality}

A mixed entangled state is a convex combination of pure states that cannot be produced by local operations and classical communication. The decomposition of a mixed entangled state (with density matrix $\rho=\sum_{k} p_{k} \rho_{1}^{k} \otimes \rho_{2}^{k} \otimes \cdots \otimes \rho_{n}^{k}$ where $0<p_{k}<1$ are the probabilities and $\rho_{i}^{k}$ is the $k$-th pure state density matrix for the $i$-th party) into the corresponding pure states $\left(\rho_{i}^{k}\right)$ is not unique 
however, and there is no direct way to extend Banik's result [22] to create games where a mixed entangled state can provide QSWS.

The above discussion raises the question:

Can mixed entangled states be used as QSWA for Bayesian games at all?

We answer this question in the affirmative, by explicitly constructing a proofof-principle Bayesian game, where a mixed entangled state gives higher unfair payoffs and higher social payoffs than classical equilibria.

\subsection{The Premise}

We consider two players Alice $(A)$ and Bob $(B)$ playing a Bayesian game while being refereed by Charlie. Both Alice and Bob have 3 possible measurement settings ( $\left\{A_{1}, A_{2}, A_{3}\right\}$ and $\left.\left\{B_{1}, B_{2}, B_{3}\right\}\right)$ which result in one of two outcomes $\{0,1\}$ each. Charlie asks $\mathrm{A}$ and $\mathrm{B}$ to implement one of these measurements each (i.e. $\left.A_{i}, B_{j}\right)$ and they reply with their respective measurement outcomes $(x, y)$. In this scenario, we define $P\left(x, y \mid A_{i}, B_{j}\right)$ to be the probability that when $A$ is asked $A_{i}$ and $B$ is asked $B_{j}$ they reply with $x$ and $y$ as their measurement outcomes, respectively, with $x, y \in\{0,1\}$.

First, we construct the classical game. Here the measurement settings can be thought of as questions asked by Charlie to Alice and Bob, and the measurement outcomes as their answers to the questions.

\subsection{The Classical Game}

\subsubsection{Classical Strategies}

A classical strategy means $A$ and $B$ both locally decide their answers to the questions. For each question, $A$ or $B$ can answer either 0 or 1 . So for the entire set of 3 questions, there are $2^{3}=8$ different sets of answers. Each such set of answers is called a strategy for that particular player. For example, if $A$ decides to answer 0 to all questions (that is 0 for $A_{1}, 0$ for $A_{2}$ and 0 for $A_{3}$ ) then her strategy is 000 .

We label these strategies $\left\{g_{i}\right\}$ by converting the binary answer sequence (for $A_{1}, A_{2}, A_{3}$ or $B_{1}, B_{2}, B_{3}$, in this order) into its decimal equivalent. For example, 000 becomes $g_{0}$ and 010 becomes $g_{2}$ and so on. The ordered pair of Alice and Bob's individual strategies $\left(g_{i}^{A}, g_{j}^{B}\right)$ called a strategy pair for $A$ and $B$.

\subsubsection{Probability Boxes}

The probability box (also called local box) is a table which shows how the strategy relates the questions to the answers. It shows for each question, with what probability a player chooses a particular answer. There is a one-to-one relation between the strategy ordered pair $\left(g_{i}^{A}, g_{j}^{B}\right)$ and the probability box.

Local boxes respect locality, that is the probability that $A$ gives a particular answer to some question is independent of what $B$ is asked and what his response is. For the sake of convenience, a general classical probability box is usually written in the form shown in Table $1 .\left\{C_{i p} M_{p}, N_{j}\right\}$ are all probabilities and hence $\in\{0,1\}$.

Row $A_{i} B_{j}$ and column xy represents probability of answering $(x, y)$ for the 
Table 1. Form of a general probability box.

\begin{tabular}{ccccc}
\hline & $O O$ & $O 1$ & $1 O$ & 11 \\
\hline$A_{1} B_{1}$ & $C_{11}$ & $M_{1}-C_{11}$ & $N_{1}-C_{11}$ & $1-M_{1}-N_{1}+C_{11}$ \\
$A_{1} B_{2}$ & $C_{12}$ & $M_{1}-C_{12}$ & $N_{2}-C_{12}$ & $1-M_{1}-N_{2}+C_{12}$ \\
$A_{1} B_{3}$ & $C_{13}$ & $M_{1}-C_{13}$ & $N_{3}-C_{13}$ & $1-M_{1}-N_{3}+C_{13}$ \\
$A_{2} B_{1}$ & $C_{21}$ & $M_{2}-C_{21}$ & $N_{1}-C_{21}$ & $1-M_{2}-N_{1}+C_{21}$ \\
$A_{2} B_{2}$ & $C_{22}$ & $M_{2}-C_{22}$ & $N_{2}-C_{22}$ & $1-M_{2}-N_{2}+C_{22}$ \\
$A_{2} B_{3}$ & $C_{23}$ & $M_{2}-C_{23}$ & $N_{3}-C_{23}$ & $1-M_{2}-N_{3}+C_{23}$ \\
$A_{3} B_{1}$ & $C_{31}$ & $M_{3}-C_{31}$ & $N_{1}-C_{31}$ & $1-M_{3}-N_{1}+C_{31}$ \\
$A_{3} B_{2}$ & $C_{32}$ & $M_{3}-C_{32}$ & $N_{2}-C_{32}$ & $1-M_{3}-N_{2}+C_{32}$ \\
$A_{3} B_{3}$ & $C_{33}$ & $M_{3}-C_{33}$ & $N_{3}-C_{33}$ & $1-M_{3}-N_{3}+C_{33}$ \\
\hline
\end{tabular}

question $A_{i} B_{j}$ that is $P\left(x, y \mid A_{i}, B_{j}\right)$. It is easy to see that this is a local box, since, for example, the probability that $A$ answers 0 to $A_{1} B_{1}$ is

$P\left(00 \mid A_{1} B_{1}\right)+P\left(01 \mid A_{1} B_{1}\right)=M_{1}$ which is the same as the probability that $A$ answers 0 to $A_{1} B_{2}$ or $A_{1} B_{3}$, etc. So the probability that $A$ given question $A_{1}$ answers 0 is the same independent of what question $B$ is asked.

\subsubsection{Utility Boxes}

For a particular game, each player chooses one out of these 8 strategies available to them. While the strategy dictates the move or answers that the player gives upon being asked the question, the reward or payoff he gets from that answer is described by the utility box. Given the strategy pair $\left(g_{i}^{A}, g_{j}^{B}\right)$, each player's individual payoffs can be calculated from the utility boxes.

$A$ 's answers are listed along the columns and $B$ s along the rows. For a question pair $\left(A_{i}, B_{j}\right)$, the ordered pair $\left(u_{1}, u_{2}\right)$ in the row $\mathrm{x}$ and column $\mathrm{y}$ of the corresponding utility box represents the payoffs $A$ and $B$ get, respectively, on answering with $x$ and $y$. We designate $A$ 's reward as $u_{1}=u_{A}\left(x, y \mid A_{i}, B_{j}\right)$ and $B$ s as $u_{2}=u_{B}\left(x, y \mid A_{i}, B_{j}\right)$. The utility boxes we use are listed below.

\begin{tabular}{ll|llll} 
& & 0 & \multicolumn{1}{c}{1} \\
\cline { 2 - 5 } For questions $A_{1} B_{1}, A_{1} B_{2}, A_{1} B_{3}$ & 0 & $\frac{2}{3}$, & 1 & $-\frac{1}{3}$, & 0 \\
& 1 & 0, & $\frac{1}{3}$ & 0, & $\frac{1}{3}$ \\
\cline { 2 - 5 } For questions $A_{2} B_{1}$ and $A_{3} B_{2}$ & 0 & $\frac{1}{2}$, & 0 & $\frac{1}{2}$, & 0 \\
& 1 & $-\frac{1}{2}$, & -1 & $\frac{1}{2}$, & 0 \\
& & & 0 & & 1 \\
For questions $A_{2} B_{3}$ and $A_{3} B_{2}$ & 0 & $-\frac{2}{3}$, & $-\frac{1}{3}$ & $\frac{1}{3}$, & $\frac{2}{3}$ \\
& 1 & $\frac{1}{3}$, & $\frac{2}{3}$ & $\frac{1}{3}$, & $\frac{2}{3}$
\end{tabular}


For questions $A_{2} B_{2}$

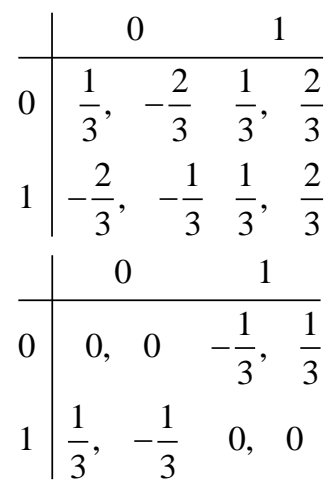

\subsubsection{Classical Payoffs}

For each strategy pair $\left(g_{i}^{A}, g_{i}^{B}\right)$ that Alice and Bob choose, they get a payoff, which can be calculated by using the strategy (probability box) to find the players answer and then using the utility box to find the corresponding payoff. The expected payoffs $\left(F_{A}, F_{B}\right)$ are then calculated by averaging over all possible questions as follows:

$$
\begin{aligned}
& F_{A}=\sum_{x, y, i, j} p(i, j) P\left(x, y \mid A_{i}, B_{j}\right) u_{A}\left(x, y, A_{i}, B_{j}\right) \\
& F_{B}=\sum_{x, y, i, j} p(i, j) P\left(x, y \mid A_{i}, B_{j}\right) u_{B}\left(x, y, A_{i}, B_{j}\right)
\end{aligned}
$$

where, $p(i, j)$ is the probability that the question pair $\left(A_{i}, B_{j}\right)$ is asked. In our game, Charlie asks Alice and Bob each question pair with equal probability. Then, $p(i, j)=\frac{1}{N^{2}} \forall(i, j)$ where $N$ is the number of questions. Here, there are 3 questions for each party so $p(i, j)=\frac{1}{9} \forall(i, j)$.

The classical payoffs, using the probability box for $P\left(x, y \mid A_{i}, B_{j}\right)$ and the utility boxes for $u_{A}$ and $u_{B}$, are then:

$$
\begin{aligned}
F_{A}= & \frac{1}{9}\left(C_{11}+C_{12}+C_{13}+C_{21}+C_{31}-C_{23}-C_{32}+C_{22}\right. \\
& \left.-M_{1}-2 N_{1}-N_{2}-\frac{M_{3}}{3}+\frac{N_{3}}{3}+2\right) \\
F_{B}= & \frac{1}{9}\left(C_{11}+C_{12}+C_{13}+C_{21}+C_{31}-C_{23}-C_{32}+C_{22}\right. \\
& \left.-M_{1}-2 N_{1}-N_{2}+\frac{M_{3}}{3}-\frac{N_{3}}{3}+3\right)
\end{aligned}
$$

The $C_{i j}, M_{i}, N_{j}$ values ( $\in\{0,1\}$ for classical strategies) are completely determined by the strategy pair $\left(g_{i}^{A}, g_{j}^{B}\right)$. Moreover, since the expressions for $F_{A}$ and $F_{B}$ are different, the payoffs are in general unfair.

\subsubsection{Classical Equilibria}

Since each of the players has a choice of 8 different strategies $\left(g_{0}, g_{1}, \cdots, g_{7}\right)$, the final payoff box is an $8 \times 8$ table (see Table 2 ) of ordered pairs, with the first entry being the payoff for Alice and the second one being the payoff for Bob. A 
Table 2. Payoff table.

\begin{tabular}{ccccccccc}
\hline & $g_{0}$ & $g_{1}$ & $g_{2}$ & $g_{3}$ & $g_{4}$ & $g_{5}$ & $g_{6}$ & $g_{7}$ \\
\hline$g_{0}$ & 6,9 & 5,10 & 6,9 & 5,10 & 3,6 & 2,7 & 3,6 & 2,7 \\
$g_{1}$ & 7,8 & $\mathbf{6 , 9}$ & 4,5 & 3,6 & 7,8 & $\mathbf{6 , 9}$ & 4,5 & 3,6 \\
$g_{2}$ & 3,6 & $-1,4$ & $\mathbf{6 , 9}$ & 2,7 & 3,6 & $-1,4$ & 6,9 & 2,7 \\
$g_{3}$ & 4,5 & 0,3 & 4,5 & 0,3 & $\mathbf{7 , 8}$ & 3,6 & $\mathbf{7 , 8}$ & 3,6 \\
$g_{4}$ & 0,3 & 2,7 & 3,6 & $\mathbf{5 , 1 0}$ & 0,3 & 2,7 & 3,6 & 5,10 \\
$g_{5}$ & 1,2 & 3,6 & 1,2 & 3,6 & 4,5 & $\mathbf{6 , 9}$ & 4,5 & $\mathbf{6 , 9}$ \\
$g_{6}$ & $-3,0$ & $-4,1$ & 3,6 & 2,7 & 0,3 & $-1,4$ & 6,9 & 5,10 \\
$g_{7}$ & $-2,-1$ & $-3,0$ & 1,2 & 0,3 & 4,5 & 3,6 & 7,8 & $\mathbf{6 , 9}$ \\
\hline
\end{tabular}

factor of $\frac{1}{27}$ has been ignored in Table 2, to keep things cleaner.

The equilibria (all are biased/unfair) have been indicated in bold font. These are the stable states for this game. Also, social welfare solution payoff is $\frac{15}{27}$. Our next task is to check whether a quantum strategy can increase payoffs of the individual parties above the classical values.

\subsection{Quantum Game}

Now, we devise the means to play this game using a quantum state. In this scenario, the two players share a Mixed Entangled State. They are asked questions $A_{1}, A_{2}, A_{3}$ and $B_{1}, B_{2}, B_{3}$ respectively, and they get their answer by performing suitable measurements on the shared state. The objective is to generate a payoff for both players that exceed the classical equilibrium payoffs.

We do this by implanting a quantum inequality in the payoff function so that quantum processes can exceed the upper bound for classical processes and hence produce payoffs higher than all classical payoffs.

\subsubsection{The Inequality}

We choose the I-3322 inequality. This inequality was discovered in 2003 by Collins and Gisin [5], but little work was done on it, other than finding it's maximal violation value using infinite dimensional quantum systems in 2010 [23].

The important thing about this inequality is that it is inequivalent to the Bell$\mathrm{CHSH}$ inequality. This means that there are states that don't violate Bell-CHSH inequality but violate this.

The inequality is usually represented in the following way:

\begin{tabular}{c|ccc} 
& -1 & 0 & 0 \\
\hline-2 & 1 & 1 & 1 \\
-1 & 1 & 1 & -1 \\
0 & 1 & -1 & 0
\end{tabular}

where the numbers correspond to the coefficients of: 


$$
\begin{array}{l|ccc} 
& P\left(A_{1}\right) & P\left(A_{2}\right) & P\left(A_{3}\right) \\
\hline P\left(B_{1}\right) & P\left(A_{1} B_{1}\right) & P\left(A_{2} B_{1}\right) & P\left(A_{3} B_{1}\right) \\
P\left(B_{2}\right) & P\left(A_{1} B_{2}\right) & P\left(A_{2} B_{2}\right) & P\left(A_{3} B_{2}\right) \\
P\left(B_{3}\right) & P\left(A_{1} B_{3}\right) & P\left(A_{2} B_{3}\right) & P\left(A_{3} B_{3}\right)
\end{array}
$$

in the expression. Here, for succinctness, we write $P\left(00 \mid A_{i} B_{j}\right)$ as $P\left(A_{i} B_{j}\right)$ and $P$ $\left(0 \mid A_{i}\right)$ as $P\left(A_{i}\right)$.

Rewriting the inequality in our chosen nomenclature, we get

$$
\begin{aligned}
S= & -\frac{1}{3} P\left(01 \mid A_{1} B_{1}\right)-\frac{2}{3} P\left(10 \mid A_{1} B_{1}\right)+\frac{1}{3} P\left(00 \mid A_{1} B_{2}\right)-\frac{1}{3} P\left(01 \mid A_{1} B_{2}\right) \\
& -\frac{1}{3} P\left(10 \mid A_{1} B_{2}\right)+\frac{2}{3} P\left(00 \mid A_{1} B_{3}\right)-\frac{1}{3} P\left(01 \mid A_{1} B_{3}\right)+\frac{1}{3} P\left(00 \mid A_{2} B_{1}\right) \\
& -\frac{2}{3} P\left(10 \mid A_{2} B_{1}\right)+\frac{2}{3} P\left(00 \mid A_{2} B_{2}\right)-\frac{1}{3} P\left(10 \mid A_{2} B_{2}\right)-P\left(00 \mid A_{2} B_{3}\right) \\
& +\frac{1}{3} P\left(00 \mid A_{3} B_{1}\right)-\frac{2}{3} P\left(10 \mid A_{3} B_{1}\right)-\frac{4}{3} P\left(00 \mid A_{3} B_{2}\right)-\frac{1}{3} P\left(10 \mid A_{3} B_{2}\right)
\end{aligned}
$$

After plugging in the variables from the probability box, Equation (2) becomes

$$
S=C_{11}+C_{12}+C_{13}+C_{21}+C_{22}-C_{23}+C_{31}-C_{32}-M_{1}-2 N_{1}-N_{2} .
$$

\subsubsection{Maximum Violation of the Inequality}

For all classical systems, I-3322 satisfies $S \leq 0$. For quantum mechanical systems however, a numerical optimization suggests that the maximum value is 0.25 [5]. The same is suggested by another approach using infinite dimensional quantum systems [23].

The state that produces this maximum value is the maximally entangled Bell state $|\bar{\psi}\rangle$

$$
|\bar{\psi}\rangle=\frac{1}{\sqrt{2}}(|01\rangle-|10\rangle)
$$

Choosing appropriate measurements for $A$ and $B$, gives the value of the inequality

$$
S_{|\bar{\psi}\rangle}=0.25
$$

Also, since $F_{A}+F_{B}=\frac{1}{9}(2 S+5)$ and this state gives the maximum possible value of $S$, this state is automatically the SWS for this game.

However, since this inequality is in-equivalent to the Bell-CHSH inequality, there exist states that violate this inequality but not the Bell-CHSH inequality. We choose one such mixed entangled state and corresponding measurements, with the aim to increase the payoffs beyond classical limits.

\subsubsection{The Quantum State}

The state shared between the $A$ and $B$ is the following mixed entangled state:

$$
\rho_{A B}=0.85|\Phi\rangle\langle\Phi|+0.15| 01\rangle\langle 01|
$$

where 


$$
|\Phi\rangle=\frac{1}{\sqrt{5}}(2|00\rangle+|11\rangle)
$$

The density matrix for the state $\rho_{A B}$ is then:

$$
\rho_{A B}=\left[\begin{array}{cccc}
0.68 & 0 & 0 & 0.34 \\
0 & 0.15 & 0 & 0 \\
0 & 0 & 0 & 0 \\
0.34 & 0 & 0 & 0.17
\end{array}\right]
$$

\subsubsection{The Measurements}

The 6 questions in the classical game have their corresponding measurements for the quantum version. These are all projective measurements, specified by their polar and azimuthal angles $(\theta, \varphi)$. The probabilities for these measurements are calculated by applying the density matrix of the proper eigenvalue of the measurement operator on the density matrix of the quantum state and then taking its trace.

$$
\begin{aligned}
& A_{1} \equiv(\eta, 0), \quad A_{2} \equiv(-\eta, 0), \quad A_{3} \equiv\left(-\frac{\pi}{2}, 0\right) \\
& B_{1} \equiv(-\chi, 0), \quad B_{2} \equiv(\chi, 0), \quad B_{3} \equiv\left(-\frac{\pi}{2}, 0\right)
\end{aligned}
$$

Such that $\cos \eta=\sqrt{\frac{7}{8}}$ and $\cos \chi=\sqrt{\frac{2}{3}}$.

Applying the measurements, with the appropriate eigenvalues, we find out all the elements of the probability box as follows:

$$
\begin{gathered}
M_{1}=0.808687, M_{2}=0.808687, M_{3}=0.5 \\
N_{1}=0.646969, N_{2}=0.646969, N_{3}=0.5 \\
C_{11}=0.576785, C_{12}=0.646188, C_{13}=0.464447, \\
C_{21}=0.646188, C_{22}=0.576785, C_{23}=0.344239, \\
C_{31}=0.421634, C_{32}=0.225335, C_{33}=0.08
\end{gathered}
$$

\subsubsection{Quantum Payoffs}

The quantum payoffs are then calculated using the same formulae as those for classical payoffs. Putting the values in Equation (1), we get:

$$
F_{A}=\frac{6.03858}{27} \text { and } F_{B}=\frac{9.03858}{27}
$$

The quantum payoff values from Equation (6), that is $\left(\frac{6.03858}{27}, \frac{9.03858}{27}\right)$ is greater than the classical equilibrium value $\left(\frac{6}{27}, \frac{9}{27}\right)$.

Also, the quantum social welfare value $\frac{15.0772}{27}$ exceeds that for all classical equilibria $\left(\frac{15}{27}\right)$. 


\section{Discussions and Conclusions}

We have, hence, constructed a game where a mixed entangled state provides higher individual payoffs than the classical equilibria. The social welfare payoff is also increased beyond the upper limit for the classical scenario. Note that the quantum strategy that we chose generated higher payoffs than one particular classical equilibrium but by modifying the utility boxes, it is possible to dominate any particular classical equilibrium without disrupting the social welfare value, thus preserving the QSWA. It is also possible to increase the social welfare value to its upper limit (strict inequality still holds) for quantum systems, $\frac{16.5}{27}$ by modifying the coefficients of the mixed entangled state. Finally, we point out that given this particular quantum advice from the referee, the measurement settings chosen maximize the Social Welfare Value and thus is a restricted SWS.

However it still remains an open question whether similar to pure entangled states, every mixed entangled state can be used as QSWA for some Bayesian game.

\section{Acknowledgements}

Authors are thankful to Prof. G. Kar, ISI, Kolkata for his guidance.

\section{Conflicts of Interest}

The authors declare no conflicts of interest regarding the publication of this paper.

\section{References}

[1] Einstein, A., Podolsky, B. and Rosen, N. (1935) Can Quantum-Mechanical Description of Physical Reality Be Considered Complete? Physical Review, 47, 777-780. https://doi.org/10.1103/PhysRev.47.777

[2] Bohr, N. (1935) Can Quantum-Mechanical Description of Physical Reality Be Considered Complete? Physical Review, 48, 696-702.

https://doi.org/10.1103/PhysRev.48.696

[3] Bell, J.S. (1987) Speakable and Unspeakable in Quantum Mechanics: Collected Papers on Quantum Philosophy. Cambridge University Press, Cambridge.

[4] Clauser, J.F., Horne, M.A., Shimony, A. and Holt, R.A. (1969) Proposed Experiment to Test Local Hidden-Variable Theories. Physical Review Letters, 23, 880-884. https://doi.org/10.1103/PhysRevLett.23.880

[5] Collins, D. and Gisin, N. (2003) A Relevant Two Qubit Bell Inequality Equivalent to the CHSH Inequality. Journal of Physics $A, 37,6$.

https://doi.org/10.1088/0305-4470/37/5/021

[6] Gibbons, R. (1992) Game Theory for Applied Economists. Princeton University Press, Princeton. https://doi.org/10.1515/9781400835881

[7] Ordeshook, P. (1986) Game Theory and Political Theory: An Introduction. Cambridge University Press, Cambridge. https://doi.org/10.1017/CBO9780511666742

[8] Colman, A. (1995) Game Theory and Its Applications: In the Social and Biological Sciences. Psychology Press, East Sussex. 
[9] Osborne, M.J. (2003) An Introduction to Game Theory. Oxford University Press, Oxford.

[10] Neumann, V. and Morgenstern, O. (1994) Theory of Games and Economic Behavior. Princeton University Press, Princeton.

[11] Nash, J.F. (1950) Equilibrium Points in n-Person Games. PNAS, 36, 48-49. https://doi.org/10.1073/pnas.36.1.48

[12] John, C.H. (1968) Games with Incomplete Information Played by "Bayesian" Players, I-III. Part II. Bayesian Equilibrium. Management Science, 14, 320-334. https://doi.org/10.1287/mnsc.14.5.320

[13] Robert, J.A. (1974) Subjectivity and Correlation in Randomized Strategies. Journal of Mathematical Economics, 1, 67-96. https://doi.org/10.1016/0304-4068(74)90037-8

[14] Nicolas, B. and Noah, L. (2013) Bell Nonlocality and Bayesian Game Theory. Nature Communications, 4, 2057. https://doi.org/10.1038/ncomms3057

[15] Taksu, C. and Azhar, I. (2008) Bayesian Nash Equilibria and Bell Inequalities. Journal of the Physical Society of Japan, 77, Article ID: 024801. https://doi.org/10.1143/JPSJ.77.024801

[16] Mermin, N.D. (1990) Quantum Mysteries Revisited. American Journal of Physics, 58, 731-734. https://doi.org/10.1119/1.16503

[17] Mermin, N.D. (1990) Simple Unified Form for the Major No-Hidden-Variables Theorems. Physical Review Letters, 65, 3373-3376. https://doi.org/10.1103/PhysRevLett.65.3373

[18] Asher, P. (1990) Incompatible Results of Quantum Measurements. Physics Letters $A, 151,107-108$. https://doi.org/10.1016/0375-9601(90)90172-K

[19] Zu, C., Wang, Y.-X., Chang, X.-Y., Wei, Z.-H., Zhang, S.-Y. and Duan, L.-M. (2012) Experimental Demonstration of Quantum Gain in a Zero-Sum Game. New Journal of Physics, 3, Article ID: 033002. https://doi.org/10.1088/1367-2630/14/3/033002

[20] Pappa, A., Kumar, N., Lawson, T., Santha, M., Zhang, S., Diamanti, E. and Kerenidis, I. (2015) Nonlocality and Conflicting Interest Games. Physical Review Letters, 114, Article ID: 020401. https://doi.org/10.1103/PhysRevLett.114.020401

[21] Roy, A., Mukherjee, A., Guha, T., Ghosh, S., Bhattacharya, S. and Banik, M. (2016) Nonlocal Correlations: Fair and Unfair Strategies in Bayesian Games. Physical Review A, 94, Article ID: 032120. https://doi.org/10.1103/PhysRevA.94.032120

[22] Banik, M., Bhattacharya, S., Ganguly, N., Guha, T., Ghosh, S., Mukherjee, A., Rai, A. and Roy, A. (2019) Bayesian Games, Social Welfare Solutions and Quantum Entanglement. Quantum, 3, 185. https://doi.org/10.22331/q-2019-09-09-185

[23] Pal, K.F. and Vertesi, T. (2010) Maximal Violation of the I3322 Inequality Using Infinite Dimensional Quantum Systems. Physical Review A, 82, Article ID: 022116. https://doi.org/10.1103/PhysRevA.82.022116 